\title{
Cloning and Expression of Two Carboxylesterases, and Their Activity Modulation in Chinese Mitten crab Eriocheir sinensis under Pesticide Exposer
}

\author{
Yanan Zang ${ }^{1}$, Xiao Zhang ${ }^{1}$, Pengdan Huang ${ }^{1}$, Kassimu Hashim Ame ${ }^{1,2}$, Huaishun \\ Shen ${ }^{1,2 *}$
}

${ }^{1}$ Nanjing Agricultural University, Wuxi Fisheries College, Nanjing, 210095, PR China.

${ }^{2}$ Ministry of Agriculture, Freshwater Fisheries Research Center, Chinese Academy of Fishery Sciences, Key Laboratory of Freshwater Fisheries and Germplasm Resources Utilization, Wuxi 214081, PR China.

\section{Article History}

Received 13 December 2018

Accepted 16 December 2019

First Online 30 December 2019

\section{Corresponding Author}

Tel.: +8618068306896

E-mail: shenhuaishun@ffrc.cn

\section{Keywords}

Carboxylesterases

cDNA clone

Chinese mitten crab

Insecticide detoxification

\begin{abstract}
Carboxylesterases (CXES) belong to a family of multifunctional enzymes. They metabolize drugs, environmental toxicants, and carcinogens, and inhibit bacterial pathogenesis. In this study, the full-length cDNAs of ES-CXE3 (2,444 bp) and ES -CXE4 $(2,385 \mathrm{bp})$ were cloned from the Chinese mitten crab, Eriocheir sinensis. Sequence analysis showed that both ES-CXE sequences contained the catalytic triplet structure characteristic of the CXES superfamily. Alignment and phylogenetic analyses revealed that the two ES-CXES are highly similar to those of other crustaceans. Tissue specificexpression analysis showed that both ES-CXES were highly expressed in the hepatopancreas. Real-time fluorescence quantitative PCR showed that the maximum expression levels of the ES-CXE3 and ES-CXE4 genes in the hepatopancreas of E. sinensis exposed to low doses of $\beta$-cypermethrin, avermectin and trichlorfon were $10 \times, 8 \times, 6 \times$ and $600 \times, 110 \times, 250 \times$ higher than relative to those of the control group, respectively, and that enzyme activities steadily increased and were significantly higher than that of the control group. Therefore, treatment with these insecticides may induce the expression of both ES-CXES as well as changes in the activities of carboxylesterase family genes. Our results suggest that ES-CXEs might play vital roles for insecticide detoxification in $E$. sinensis
\end{abstract}

\section{Introduction}

Carboxylesterases (CXES) are ubiquitous aliphatic esterases in animals, plants, and microorganisms (Jeon et al., 2011). They have a catalytic triad structure and a near- $N$-terminus glycosylation site, which maintains enzyme activity and stabilizes the active sites (Zhang, 2014). Carboxylesterases belong to a superfamily of multifunctional enzymes that participate in signal transmembrane transduction, metabolic detoxification of organophosphorus insecticides and other pest control products, and lipid synthesis and decomposition (Teng and Sun, 2003; Zhang et al., 2012).In insects, insect resistance to organophosphorus, carbamate, and deltamethrin is correlated with the in vivo enhancement of the metabolic activity of CXEs, achieved mainly by CXE gene amplification, regulation of CXE gene expression, and CXE gene mutation (Li et al., 2007; Dou et al., 2010; Grigoraki et al., 2016).

Eriocheir sinensis is also known as the river crab and it is an economically important crustacean cultivated in China (Shen et al., 2017). With the rapid increase of the $E$. sinensis aquaculture industry, numerous diseases have recently evolved (Shen et al., 2015). Both abiotic and biotic stressors are intensifying in aquaculture. Numerous diseases have recently evolved and many pest control products have been used (Geng, 2010). In agricultural production, insecticides are used to kill crop pests. Pesticide residues may enter aquatic ecosystems via surface runoff, rainwater scour, 
and domestic wastewater. These inputs may also pollute aquaculture water sources (Xu and Liu, 2017). Pesticide residues might therefore cause various crustacean diseases. Trichlorfon is often used as an agricultural pesticide, in order to control parasites on the surface of aquatic (Chang et al, 2010); avermectin has good effect on parasite control of shrimp and crab (Kovecses et al, 2002); Beta-cypermethrin is a commonly used pyrethroid insecticide. It is mainly used in aquaculture to clear algae in ponds and kill parasites on the surface of crustaceans (Wendt-Rasch et al, 2003). The three insecticides are less toxic to mammals, but are extremely toxic to aquatic animals (Tatjana et al, 2006). As an important detoxification enzyme, there are litter known about pesticide resistance mechanisms of CXES in Eriocheir sinensis. Based on the study of insect CXES gene in the metabolism and detoxification of pesticides and Shen et al. (2017) on the up-regulation of carboxylesterase gene expression in Chinese mitten crabs with hepatic pancreatic necrosis (HPND), the two carboxylesterase sequences obtained from the transcriptome data of the laboratory were studied for their molecular characteristics and expression patterns, which laid a foundation for studying the mechanism of metabolic detoxification.

\section{Materials and Methods}

\section{Experimental Animals and RNA Isolation}

Chinese mitten crabs were obtained from a breeding pond in Yandu District, Yancheng City, Jiangsu Province, China, and raised in a container $(97 \times 48 \times 63$ $\mathrm{cm}$ ) equipped with a pump oxygen system to simulate their natural growth environment. The animals received commercial feed and water temperature was maintained at $20.0 \pm 1.0{ }^{\circ} \mathrm{C}$. Before the onset of the experiment, the animals were maintained in the container for 1 week to become acclimated to the environment.

Total RNAs were extracted from tissue samples using TRIzol reagent (Beijing Cwbiotech Company, Beijing, China) according to the manufacturer's instructions. While RNA integrity was verified by electrophoresis on a $1.5 \%$ agarose gel, RNA purity was quantified by reading its absorbance at 260 and $280 \mathrm{~nm}$ $\left(\mathrm{OD}_{260 / 280}\right)$. The RNAs with $\mathrm{OD}_{260 / 280}=1.8-2.2$ were stored at $-80^{\circ} \mathrm{C}$ until use in subsequent analyses.

\section{Cloning of Full-length CXE cDNAs}

The full-length cDNAs of two ES-CXEs were obtained by rapid amplification of CDNA ends (RACE) according to the SMARTer ${ }^{\circledR}$ RACE 5' Kit User Manual and 3'-Full RACE Core Set v. 2.0 (TaKaRa Bio Inc., Kusatsu, Shiga, Japan). Partial CDNA sequences putatively encoding ES-CXES were obtained from previously collected hepatopancreas transcriptome data (Shen et al., 2017). Gene-specific primers were designed using these partial cDNA sequences (Table 1). The final PCR products were purified using a gel extraction kit (XWBIO, Beijing, China), ligated into a pMD19-Tvector (TaKaRa Bio Inc.), and transformed into competent Escherichia coli cells. The positive transformants were selected and sequenced in both directions. The sequencing results were used to assemble full-length cDNA sequences of the ES-CXEs.

\section{Bioinformatics Analysis}

The open reading frame (ORF) finder (http://www.ncbi.nlm.nih.gov/gorf/gorf.html) was used to search for ORFs in the obtained two sequences. The NCBI basic local alignment search tool (BLAST) was used to verify the similarity of the deduced amino acid sequences of the two CXEs (http://blast.ncbi.nlm.nih.gov/). The molecular mass and isoelectric point $(\mathrm{PI})$ of each CXE were estimated by the Compute $\mathrm{PI} / \mathrm{Mw}$ tool (http://www.expasy.ch/tools/pi_tool.html). Amino acid multiple sequence alignment was performed using DNAMAN v. 5.2.2 and multiple sequence comparison tools (http://www.bio-soft.net/sms/). A phylogenetic tree was constructed in MEGA v. 6.0 using the maximum likelihood $(\mathrm{ML})$ method, based on the putative amino acid sequences of the identified CXEs and other related CXEs, and branch support was based 1,000 bootstrap repetitions.

\section{CXE mRNA Expression in Various Tissues}

Three vigorous, healthy, mature crabs of both sexes were used to determine the tissue distributions of the identified ES-CXEs. Samples of the following tissues were collected from each crab for RNA isolation: hepatopancreas, gills, heart, muscle, intestine, accessory sex gland, ovary, and seminal vesicle. The tissues were freshly dissected, frozen in liquid nitrogen, and stored at $-80^{\circ} \mathrm{C}$ until used for total RNA extraction.

\section{Induced CXE Expression and Enzyme Activity under Three Different Pesticide Treatments}

Twenty -five healthy and vigorous crabs, each weighing $5.5 \pm 0.5 \mathrm{~g}$, were randomly assigned to one of four treatment groups ( $\beta$-cypermethrin, avermectin, trichlorfon and no pesticide added; further information on these three pesticides is given in Table 2), each with three replicates, and placed into one of 12 different containers $(97 \times 48 \times 63 \mathrm{~cm})$. The concentrations of the three aforementioned pesticides, diluted with $\mathrm{ddH}_{2} \mathrm{O}$, were $0.002 \mu \mathrm{g} / \mathrm{L}, 0.05 \mathrm{~g} / \mathrm{L}$, and $0.001 \mu \mathrm{g} / \mathrm{L}$, respectively, in each container. The concentration set for each of the insecticides was based on the safe concentration obtained from laboratory semi-lethal concentration experiments $\left(8.52 \times 10^{-5} \mu \mathrm{g} / \mathrm{L}\right.$ for $\beta$-cypermethrin, $4.08 \times 10^{-4} \mu \mathrm{g} / \mathrm{L}$ for avermectin and $5.00 \times 10^{-4} \mathrm{~g} / \mathrm{L}$ for trichlorfon). Three crabs from each container were 
Table 1 Primers used in the present study

\begin{tabular}{lcc}
\hline Primers & Sequence $\left(5^{\prime}-3^{\prime}\right)$ & Primer description \\
\hline ES-CXE3-5'R1 & TGCACCACCACCAGTACCACGTCGT & 5' RACE primer for first round \\
ES-CXE3-5'R2 & CTCCTGTAGCCACCACCACCATCGC & 5' RACE primer for second round \\
ES-CXE3-3'F1 & CAGAAAGAGCCAGCAGAAGGA & 3' RACE primer for first round \\
ES-CXE3-3'F2 & GTCGTAGTAACTTAGCTGGTG & 3' RACE primer for second round \\
ES-CXE4-5'R1 & TGACGGGGCTTCGGGGTCCTTGAA & 5' RACE primer for first round \\
ES-CXE4-5'R2 & CGCCCCTTCAACGTTTTCTCCAGG & 5' RACE primer for second round \\
ES-CXE4-3'F1 & CTAATCAGACATCTTGAGGC & 3' RACE primer for first round \\
ES-CXE3-R & GAGCGTCAGGAAGGGGAAACC & RVS primer for ES-CXE3 expression \\
ES-CXE3-F & GAGTCATGACCTCACCACCGC & FWD primer for ES-CXE3 expression \\
ES-CXE4-R & GTTGGTCCAGAGCGTCGTCA & RVS primer for ES-CXE4 expression \\
ES-CXE4-F & GCGACCGAGAGTGGGTGAAC & FWD primer for ES-CXE4 expression \\
$\beta-$-actin-R & CTCCTGCTTGCTGATCCACATC & RVS primer for $\beta$-actin expression \\
$\beta$-actin-F & GCATCCACGAGACCACTTACA & FWD primer for $\beta$-actin expression \\
\hline
\end{tabular}

Table 2 Information on the three tested insecticides

\begin{tabular}{lccc}
\hline Insecticide & Company & Concentration & Registration number \\
\hline Avermectin & Shanxi Shouai Animal Pharmaceutical industry & $1 \%$ & PD20040372 \\
Trichlorfon & Nantong Jiangshan pesticide chemical industry & $90 \%$ & Veterinary drug GMP No. 04031 \\
$\beta$-cypermethrin & Zhejiang Welda chemical industry & $4.5 \%$ & PD84108-5 \\
\hline
\end{tabular}

sacrificed at $0,3,6,12,24$ and $48 \mathrm{~h}$ after pesticide administration. The hepatopancreas of each crab was collected for RNA isolation and CXEs family activity assays.

\section{Real-time Fluorescence Quantitative PCR}

The mRNA expression patterns of the cloned ESCXEs in the various tissues and their expression levels at different time points after pesticide treatment were examined by real-time fluorescence quantitative PCR (qRT-PCR), using primers (Table 1 ) designed according to the full-length cDNAs of ES-CXEs. The internal reference gene 8 -actin (GenBank Accession no. HM053699.1) was used to calibrate the cDNA template. The qRT-PCR was performed with the $A B I 7500$ system (Applied Biosystems, Foster City, CA, USA). The reaction conditions were as follows: denaturation at $95^{\circ} \mathrm{C}$ for 10 min; 40 cycles of $95^{\circ} \mathrm{C}$ for $15 \mathrm{~s}$; and $60^{\circ} \mathrm{C}$ for $1 \mathrm{~min}$.

Three replicates were prepared for each sample. The comparative $\mathrm{Ct}$ method $\left(2^{-\Delta \Delta \mathrm{t}}\right)$ as described by Livak (2008) was used to calculate the relative expression of each target CXE gene in different tissues and in the hepatopancreas after pesticide treatment. Specificity of the amplification for all target genes and 6 -actin was confirmed by a melting curve analysis performed on SDS software (Applied Biosystems Inc., Foster City, CA, USA). Data were statistically analyzed with SPSS v. 18.0 (IBM Corp., Armonk, NY, USA) using single-factor analysis of variance (ANOVA) considering $P<0.05$ as the significance threshold. Data are expressed as means \pm standard deviation.

\section{CXE Family Activity Assays}

The activities of CXE family genes at different time points after pesticide treatment were determined by spectrophotometry using the Carboxylesterase Activity Assay Kit (Beijing Solarbio Company, Beijing, China) and the method measured and activity calculation was performed according to the Lai, et al (2018). Data were statistically analyzed with SPSS v. 18.0 (IBM Corp.) using single-factor ANOVA and considering $P<0.05$ as the significance threshold. Data are expressed as means \pm standard deviation.

\section{Statistical Analysis}

The statistical analysis was performed using SPSS v. 18.0 (IBM Corp.) (* indicated $P<0.05, * *$ indicated $P<$ 0.01). Data are expressed as means \pm standard deviation, and the sampling points for the different treatments were analyzed by using single-factor ANOVA.

\section{Results}

\section{cDNA Cloning of CXEs}

Full-length of two ES-CXE cDNAs were isolated from the hepatopancreas of the Chinese mitten crab. Because two juvenile hormone esterase-like (JHE-like) CXE genes have been reported (Xu et al., 2017), the two $E S-C X E$ genes cloned in the present study were named ES-CXE3 and ES-CXE4. Sequence analysis revealed that 
the full-length cDNA ES-CXE3 sequence obtained from the hepatopancreas of Chinese mitten crab by RACE was 2,446 bp (GenBank Accession No. MH201556). It consisted of a $5^{\prime}$-untranslated region (UTR) of $150 \mathrm{bp}$, a 3'-UTR of 526 bp with a polyadenylation signal (AATAA) and a Poly-A tail, and an ORF of 1,770 bp. This ORF encoded 589 amino acids with an estimated mass of $65.38 \mathrm{kDa}$ and a predicted PI of 5.45 (Supplement Figure S1 (a)). The full-length cDNA sequence of ES-CXE4 was 2,384 bp (GenBank Accession No. MH291557). It consisted of a 5'-UTR of $72 \mathrm{bp}$, a 3'-UTR of $536 \mathrm{bp}$ with a polyadenylation signal (AATAA) and a Poly-A tail, and an ORF of 1,776 bp encoding 591 amino acids with an estimated mass of $65.09 \mathrm{kDa}$ and a predicted PI of 4.79 (Supplement Figure S1 (b)). The amino acid identity between ES-CXE3 and ES-CXE4 was 74\%.

\section{Aminoacid Homology and Phylogenetic Relationships}

The deduced amino acid sequences of the two ESCXEs were aligned with related CXEs derived from several insect and crustacean species. Multiple alignments revealed that both ES-CXES contained domains typical of the CXE family proteins (Thomas et al., 1999), including three amino acid residues of the catalytic triad serine (S), glutamic acid (E), and histidine (H), RF and GG regions, and a catalytic $N$-terminus region. A carboxylesterase-specific glycine $(G) \times S \times G$, which includes the $S$ residue of the catalytic triad, was conserved in both ES-CXE3 and ES-CXE4 (Figure 1). Alignment and phylogenetic analyses revealed that the amino acid identity between ES-CXE3 and Portunus trituberculatus was the highest, about $47.97 \%$. The amino acid identity between ES-CXE4 and Portunus trituberculatus was the highest, about $48.07 \%$.

The evolutionary relationships between these two ES-CXES and those from insects and other crustaceans were evidenced in the phylogenetic tree constructed based on the multiple amino acid sequence alignment. This phylogenetic tree showed that the two ES-CXES belonged to the same crustacean CXE group as the JHElike CXE proteins from Pandalopsis japonica, Neocaridina denticulata, and Portunus trituberculatus (Figure 2).

\section{Tissue Distribution of ES-CXES}

Relative expression levels obtained from the qRTPCR used to test the tissue distribution of the ES-CXES (Figure 3) indicated that ES-CXE3 was highly expressed in the hepatopancreas, muscle, testes, and accessory gonadal glands. However, its expression levels were low in the heart, gills, and ovaries. Although ES-CXE4 was also prominently expressed in the hepatopancreas, its expression levels were nearly zero in the heart, gills, and ovaries. Generally, ES-CXE3 expression levels were higher than those of ES-CXE4 in the testes and accessory gonadal glands.

\section{ES-CXE Expression Pattern Analysis after Pesticide Treatment}

Induction of ES-CXE expression was determined in the hepatopancreas following exposure to $\beta$ cypermethrin, avermectin, or trichlorfon (Figure 4). The expression levels of both ES-CXEs significantly increased in the hepatopancreas following pesticide treatment. Twelve hours after the $\beta$-cypermethrin treatment, ESCXE3 and ES-CXE4 expression levels were $10 \times$ and $600 \times$ higher in the experimental group than in the control group, respectively. Twenty-four hours after the avermectin treatment, ES-CXE3 and ES-CXE4 expression levels were $8 x$ and $110 x$ higher in the experimental group than in the control group, respectively. Six hours after the trichlorfon treatment, ES-CXE3 and ES-CXE4 expression levels were $4 \times$ and $250 x$ higher in the experimental group than in the control group, respectively.

\section{Analysis of CXEs Family Activity Change Patterns after Pesticide Treatment}

The enzyme activities of the CXEs in the hepatopancreas determined following exposure to $\beta$ cypermethrin, avermectin, or trichlorfon (Figure 5, because there was no evident change in patterns at the six time points, no data is presented for the blank group) were significantly higher than that of the control group. The highest activities of ES-CXEs in the hepatopancreas under the $\beta$-cypermethrin, avermectin, or trichlorfon, were $8 x, 9 x$, and $6 x$ that of the control group, respectively.

\section{Discussion}

In the present study, two cDNAs encoding ES-CXEs were cloned from $E$. sinensis in our laboratory according to a transcriptome database. Multiple alignment analysis revealed that both ES-CXEs contain motifs typical of the CXE family proteins (Thomas et al., 2015; Xu et al., 2017). Previous studies proposed that JHE-like CXEs from $P$. trituberculatus, $P$. japonica, E. sinensis and $N$. denticulata have esterase activity (Lee et al., 2011; Sin et al., 2015; Tao et al., 2017; Xu et al., 2017). Multiple alignment analysis indicated that the sequences of ESCXE3 and ES-CXE4 resemble those of the JHE-like CXEs. Therefore, ES-CXE3 and ES-CXE4 might have esterase activity.

Based on sequence similarities and substrate specificities, insect CXEs with catalytic activity can be assigned to five subfamilies: $\alpha$-esterases, $\beta$-esterases, JHEs, acetylcholinesterases, and integument esterases (Oakeshott et al., 2005). In the present study, however, ES-CXE3 and ES-CXE4 genes cloned from E. sinensis, and JHE-like CXEs from other crustaceans, were classified as crustacean CXEs. This classification differs from existing traditional ones and suggests a new CXE race. According to the phylogenetic tree, crustacean CXEs were 


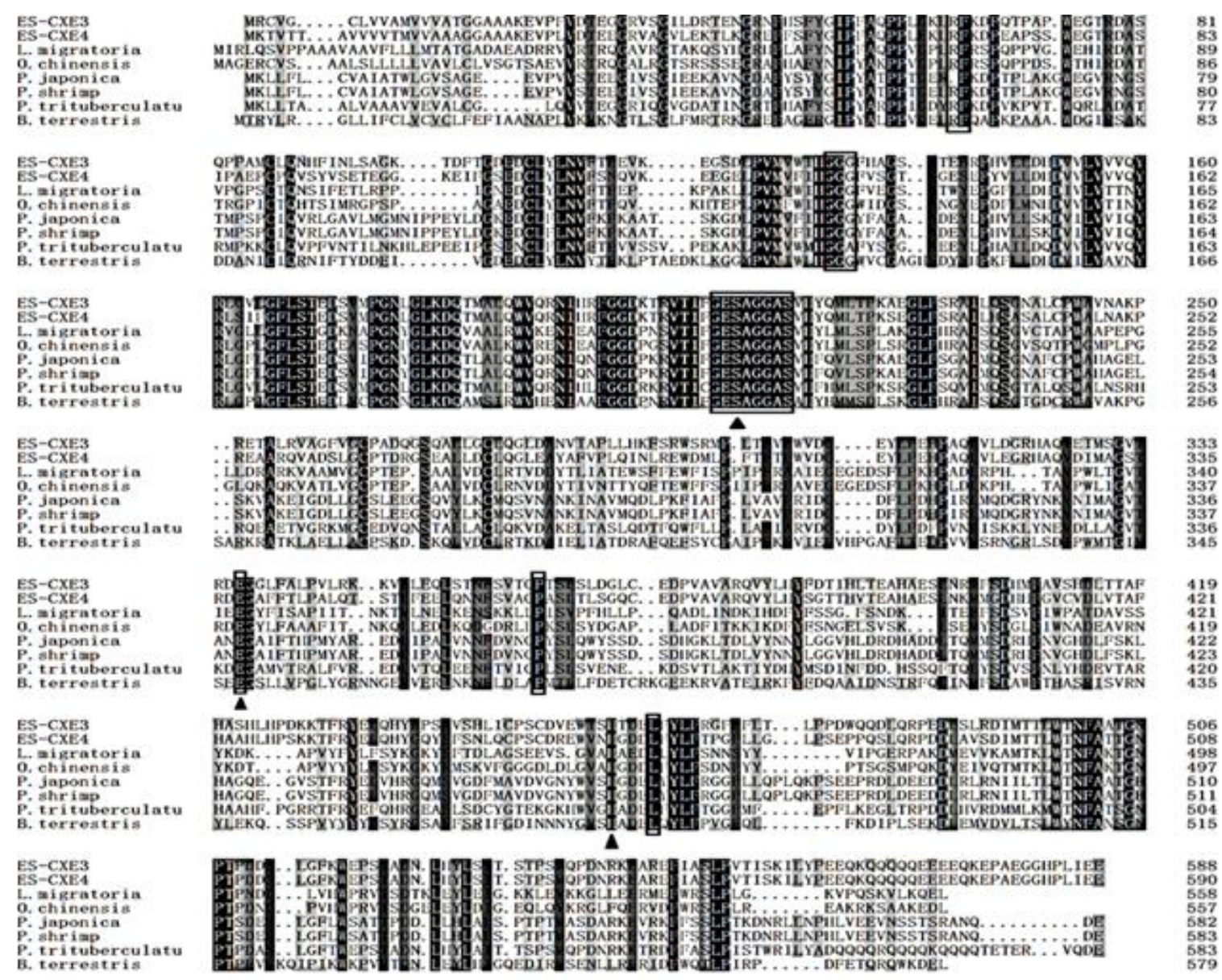

Figure 1. Multiple alignment of ES-CXE3 and ES-CXE4 amino acid sequences from a selection of related species. GenBank accession numbers by species: Pandalus borealis (HQ406776), Portunus trituberculatus (ALT10384.1), Pandalopsis japonica (ADZ9996217.1), Locusta migratoria (AHJ81347.1), Oxya chinensis (AJP62564), Bombus terrestris (XP003399739.1), and Eriocheir

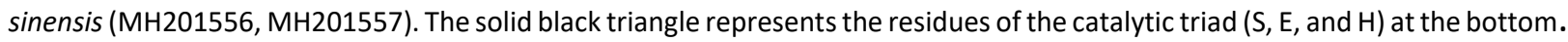
Boxes indicate residues or motifs characteristic of carboxylesterases.

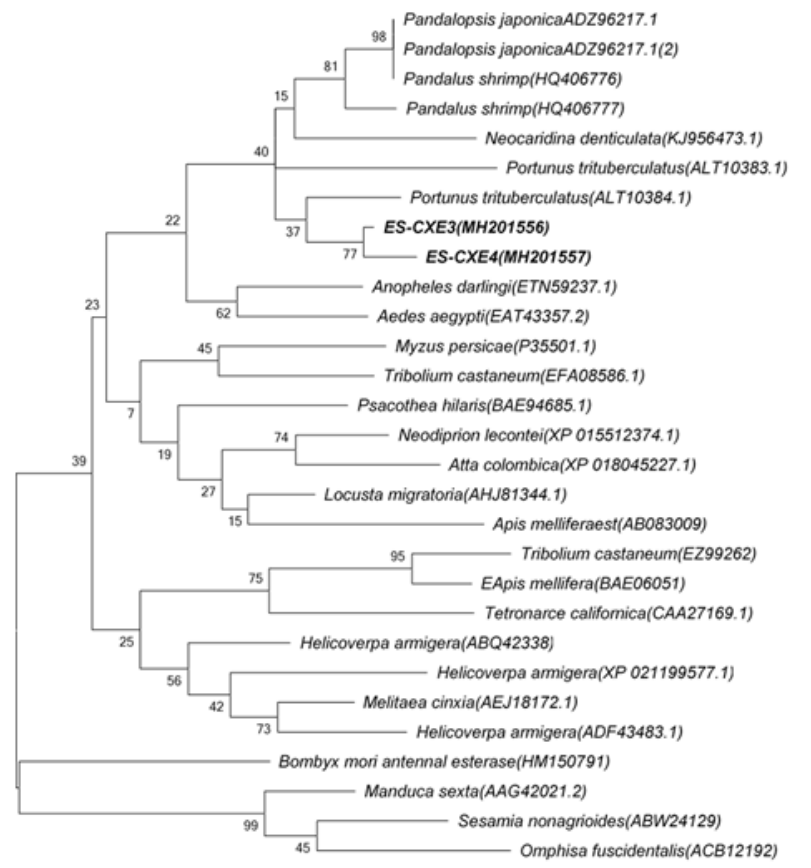

Crustacean CXE

Nonlepidopteran JHEs

$\beta$-esterases

Acetylcholin

$\alpha$-Esterases

Lepidopteran JHEs

Figure 2. Phylogenetic analysis of the deduced ES-CXEs amino acid sequences relative to other carboxylesterases. MEGA v. 6.0 was used to construct the phylogenetic tree, based on maximum likelihood and using 1,000 bootstrap replications. GenBank accession numbers are shown in the tree. 

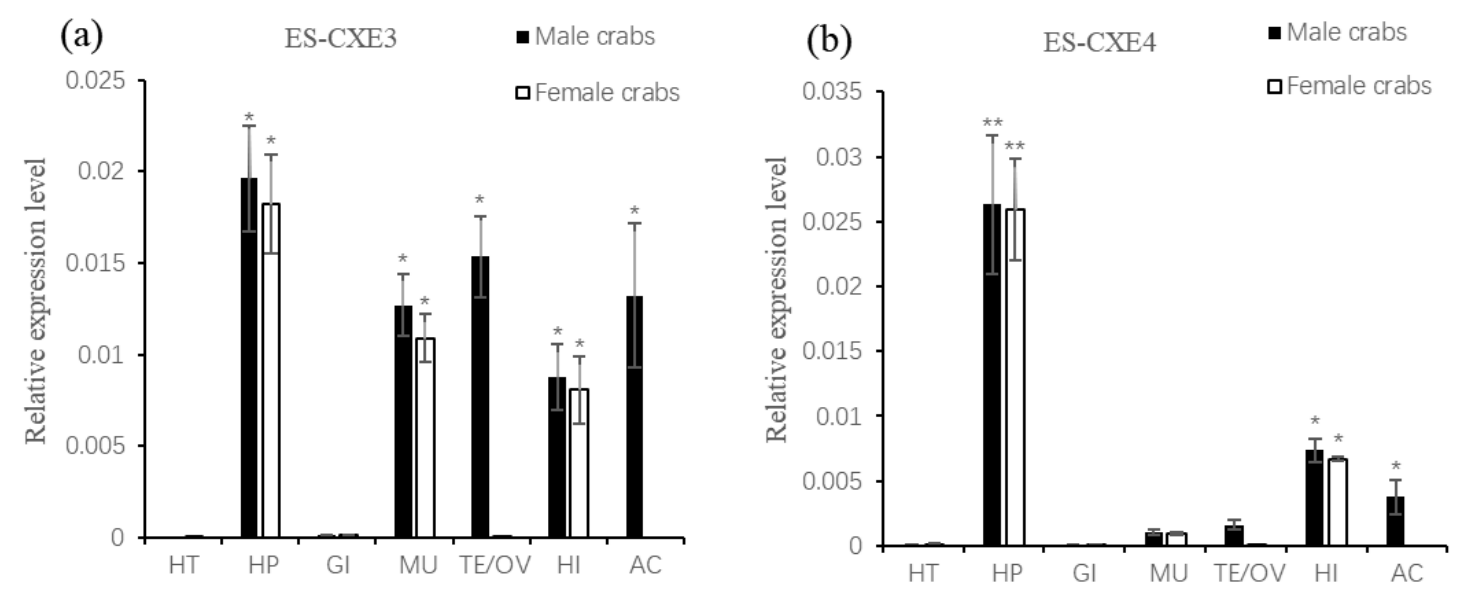

Figure 3. Tissue distribution analysis of ES-CXE3 (a) and ES-CXE4 (b). Relative expressions were normalized to the 8-actin reference gene. $\mathrm{HT}$, heart; $\mathrm{HP}$, hepatopancreas; GI, gill; MU, muscle; TE, testis; $\mathrm{HI}$, hindgut; OV, ovary; $\mathrm{AC}$, accessory gonadal gland. Bars represent mean \pm standard error of the mean $(\mathrm{n}=3)$. Asterisks represent values statistically different $\left({ }^{*} P<0.05,{ }^{* *} P\right.$ $<0.01)$.
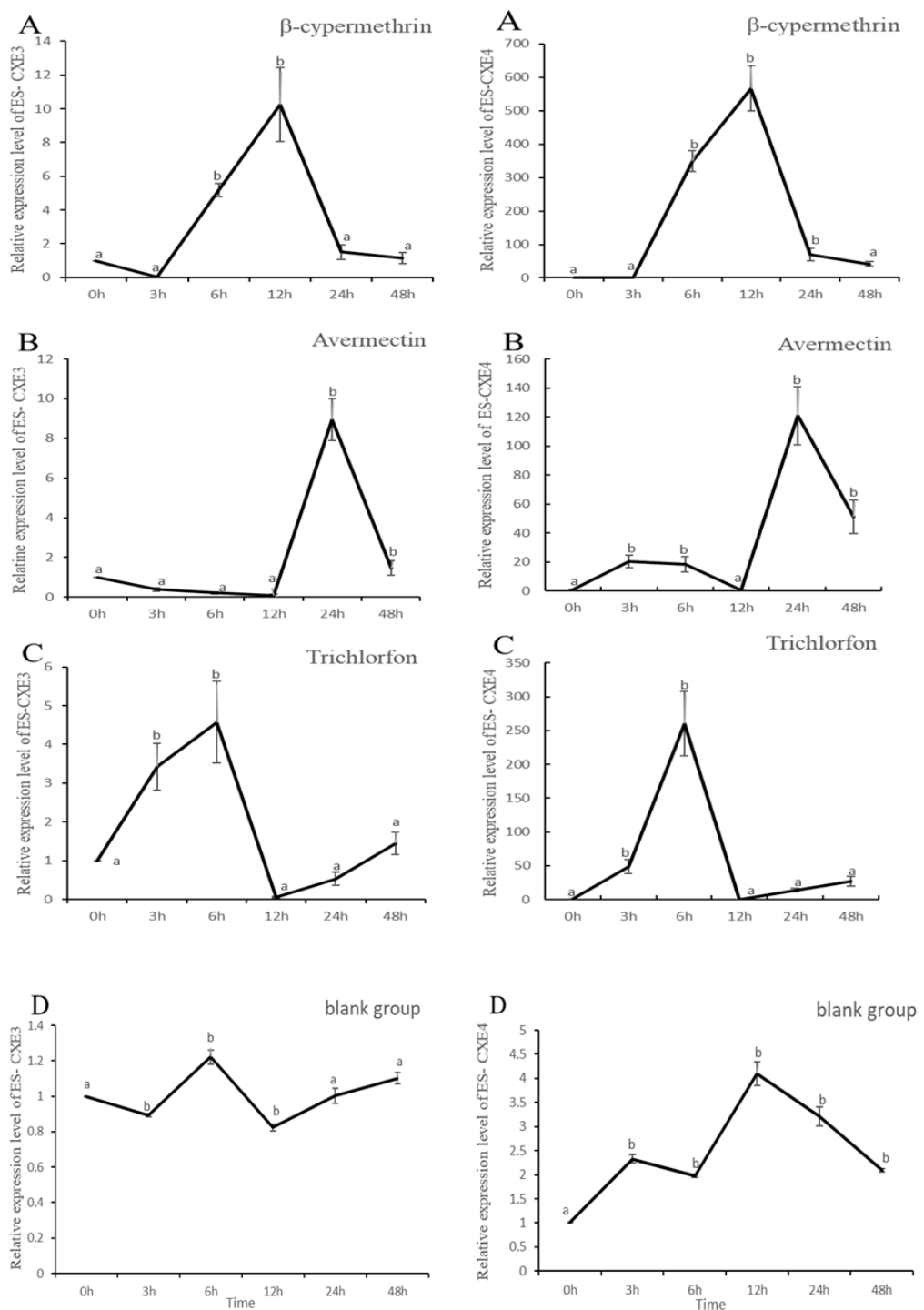

Figure 4. qRT-PCR analysis of the relative ES-CXEs expression levels in the hepatopancreas after treatment with $\beta$-cypermethrin $(A)$, avermectin $(B)$, and trichlorfon $(C)$, blank(D) (mean \pm standard error of the mean; $n=3$ ). The $B$-actin gene expression was used as an internal control. ES-CXE expression levels determined at the first time point were used as references. Means with different lowercase letters are significantly different $(P<0.05)$. 

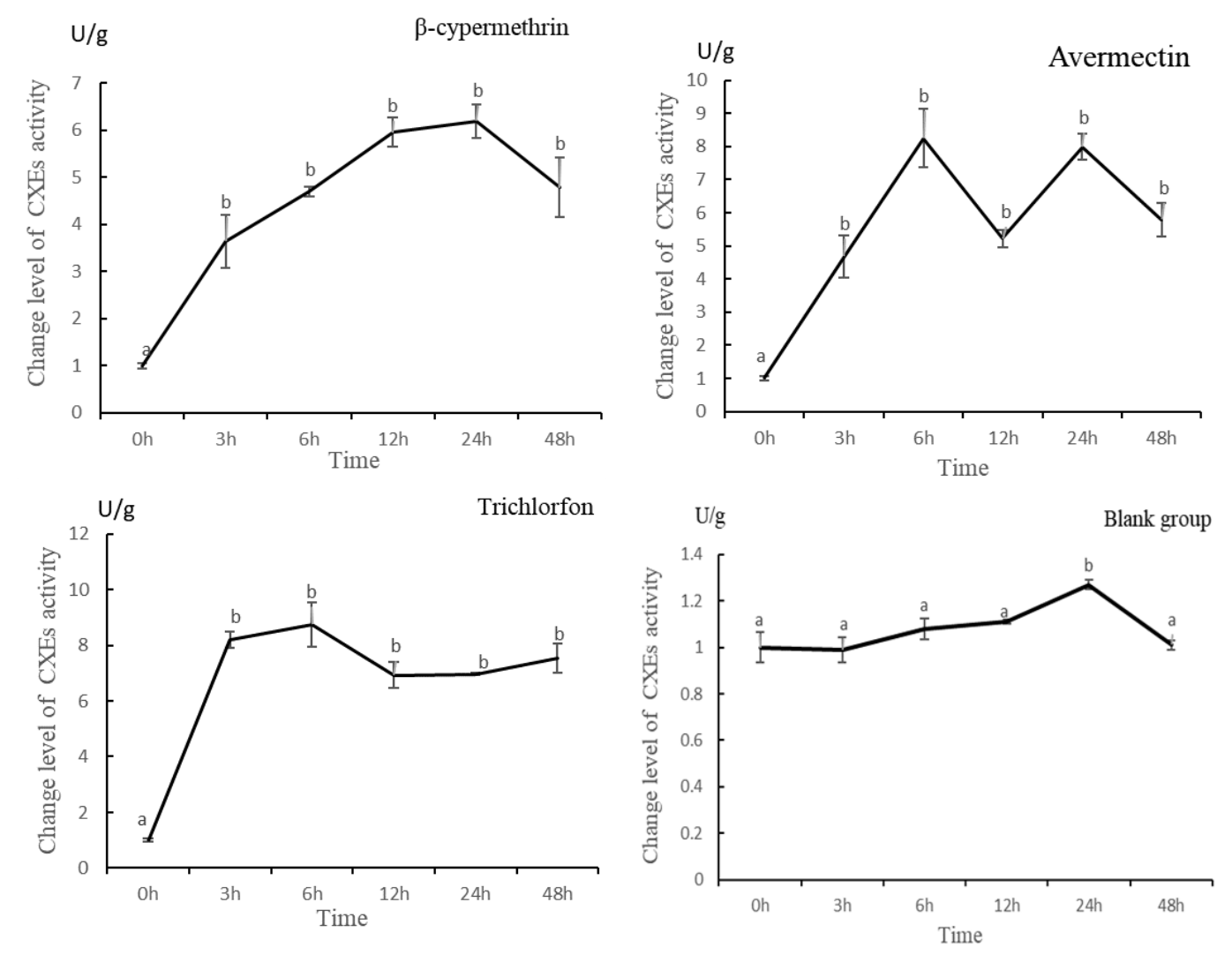

Figure 5. Carboxylesterase activity patterns in the hepatopancreas after treatment with $\beta$-cypermethrin, avermectin, trichlorfon, and blank group (mean \pm standard error of the mean; $n=3$ ). Means with different lowercase letters are significantly different $(\mathrm{P}<0.05)$.

clustered with the $\beta$-esterases and non-lepidopteran JHEs from insects. Previous studies showed that $\beta$ esterases mediate the metabolism of many pesticides and other heterologous substances (Oakeshott et al., 2005). In addition, JHE genes have been associated with the development and application of late-model insecticides (Ren et al., 2014). Therefore, the two ESCXEs might mediate insecticide metabolism.

Previous research has shown that the CXEs from $P$. trituberculatus, $P$. japonica, N. denticulata, and $E$. sinensis participate in hormone metabolism (Lee et al., 2011; Tao et al., 2017; Xu et al., 2017). The phylogenetic tree obtained here evidenced that JHE-like CXES clustered into a crustacean CXEs group along with the two ES-CXES analyzed in the present study, but a systematic classification of crustacean CXES is still needed. In addition, two full-length ES-CXE DNA sequences have been previously cloned and characterized (Xu et al., 2017) to validate the probable function in pheromone and $\mathrm{JH}$ degradation. Although it is not indicated in the phylogenetic tree, all four ES-CXES belong to the crustacean ES-CXES group.

Studies on insect CXES have revealed that lipid bodies are the main sites for protein metabolism and enzyme synthesis. The main functions of lipid bodies are energy storage and detoxification (Arrese and Soulages, 2010; Zhang, 2014). Cytochrome P450, glutathione Stransferase, and CXEs, the three major detoxifying enzymes in insects (Taylor and Radic, 1994), are all highly expressed in lipid bodies (Arrese and Soulages, 2010). The hepatopancreas of Crustacea resembles insect lipid bodies, as it is the main site for the metabolism of endogenous and exogenous compounds (Lima, 2013; Tao et al., 2017). Therefore, the hepatopancreas might be the major tissue source of crustacean ES-CXES. This hypothesis was confirmed in studies of $P$. trituberculatus, $P$. japonica, and $E$. sinensis (Ren et al., 2014; Tao et al., 2017; Xu et al., 2017). The present study showed that the expression levels of the ES-CXES were higher in the hepatopancreas than in other tissues. In P. trituberculatus and P. japonica, the ovaries also present high CXE expression levels (Lee et al., 2011; Tao et al., 2017). However, in the present study, ES-CXE3 and ES-CXE4 were only slightly expressed in the ovaries. Moreover, the relative expression of ESCXE3 was more widespread than that of ES-CXE4. Therefore, ES-CXE3 might have more metabolic functions than ES-CXE4.

Carboxylesterase mediates insecticide resistance by increasing the hydrolysis of these substances, creating barriers, or altering their enzyme affinities (Li et al., 2007; Lima, 2013). Increases in CXE mRNA expression levels may enhance enzyme activity and, consequently, insecticide resistance. Moreover, this higher activity of CXES in the body also enhances detoxification and the metabolism of exogenous 
compounds, and the resistance to insecticides (Feng et al., 1999; Liu et al., 2015). In the present study, the expression levels of the two ES-CXES were higher in the hepatopancreas of $E$. sinensis exposed to the insecticides than in the control group, the tested insecticides induced ES-CXE3 and ES-CXE4 expression. Moreover, the activities of CXEs increased steadily with exposure time. Therefore, the two ES-CXEs identified in our transcriptome analysis are involved in the detoxification of three pesticides, which have metabolic detoxification effects. Comparing the relative expression level of two ES-CXES and the trend of esterase activity, it can be seen that the change of expression amount shows a tendency to fluctuate up and down, while the activity of enzyme enzymes basically keeps rising, presumably due to the stimulation by insecticides. The interaction between genes, through mutual stimulation and inhibition, plays a role in continuous regulation, and the increase in the expression of CXEs gene will increase the detoxification enzyme activity of carboxylesterase, thus showing a continuous upward trend. Whatever, based on the changes observed on the activity of CXEs, these enzymes are more resistant to trichlorfon than to other insecticides. In addition, the expression level of ES-CXE4 was significantly higher than that of ES-CXE3. Therefore, ES-CXE4 might play a more important role in metabolic detoxification than ES-CXE3. Infection of the freshwater Chinese mitten crab E. sinensis with HPND has been a major problem in the crab-cultivation Chinese Province of Jiangsu since 2015. Hepatopancreatic injury caused by environmental toxicants is believed to be one of the main causes of HPND. However, the etiology of HPND is unknown. In our previous study (Shen et al., 2017), the expression level of the ES-CXE gene was significantly higher in $E$. sinensis with HPND than in $E$. sinensis without HPND, which is in line with the results obtained in the present study. Taken together, the results of the present and previous related studies indicate that pesticide use might be associated with crab HPND during $E$. sinensis breeding.

In summary, full-length sequences of two ES-CXE genes from Chinese mitten crab were cloned and characterized, and tissue-specific expression levels shows both ES-CXES were highly expressed in the hepatopancreas. treatment with these insecticides may induce the expression of both ES-CXEs as well as changes in the activities of carboxylesterase family genes. We believe that this study will provide insight on the pesticide resistance mechanisms associated with the CXEs in Chinese mitten crab.

\section{Acknowledgements}

Funding: This work was supported by the Natural Science Foundation of Jiangsu Province (BK20181138); the Aquatic Three Update Project of Jiangsu Province (Y2016-35); the Central Public Interest Scientific
Institution Basal Research Fund of the Freshwater Fisheries Research Center, CAFS (2017JBFM01).

\section{References}

Arrese, E. L., \& Soulages, J. L. (2010). Insect fat body: energy, metabolism, and regulation. Annual Review of Entomology. 55, 207-225. http//doi:10.1146/ annurevento- 112408-085356

Chang, Ch. Ch., Lee, P. P., Liu, Ch. H., Cheng, W. T. (2006). Trichlorfon, an organophosphorus insecticide, depresses the immune responses and resistance to Lactococcus garvieae of the giant freshwater prawn Macrobrachium rosenbergii. Fish \& Shellfish Immunology. 20, 574-585. http://doi:10.1016/j.fsi.2005.06.012

Dou, W., Xiao, L. S., Niu, J. Z., Jiang, H. B., \&Wang, J. J. (2010). Characterization of the purified glutathione Stransferades from two psocids Liposcelis bostrychophila and L. entomophila. Agricultural Sciences in China. 9 (7), 1008-1016. http// doi: 10.1016/S1671-2927(09)60184-X

Feng, Q. L., Ladd, T. R., Tomkins, B. L., Sundaram, M., Sohi, S. S., Retnakaran, A., Davey K.G., \& Palli, S. R. (1999). Spruce budworm (Choristoneura fumiferana) juvenile hor- mone esterase: hormonal regulation, developmental expression and cDNA cloning. Molecular and Cellular Endocrinology. 148 (1), 95-108. http// doi: 10.1016/S0303-7207(98)00228-7

Geng, X. (2010). Studies on detection method and toxicity of deltamethrin in river crabs, Eriocheir sinensis. (MD Thesis). Nanjing Agricultural University, Nanjing, China.

Grigoraki, L., Balabanidou, V., Meristoudis, C., Miridakis, A., Ranson, H., \& Swevers, L. (2016). Functional and immunohistochemical characterization of cceae $3 a$, a carboxyle- sterase associated with temephos resistance in the major arbovirus vectors aedes aegypti and ae. albopictus. Insect Biochemistry and Molecular Biology, 74, 61-67. http//doi:10.1016/j.ibmb.2017.05.003

Jeon, J., Kim, S., Lee, H., Cha, S., Lee, J., Yoon, S., Koo, B., Lee, C., Choi, S., \& Kang, S. (2011). Novel metagenomederived craboxylesterase that hydrolyzes $\beta$-lactam antibiotics. Applied and Environmental Microbiology. 77, 7830-7836.

Kovecses, J. (2002). Avermectins: Potential environmental risks and impacts on freshwater ecosystems in Quebec. Fibers \& Polymers. 11(3), 455-463. http://doi:10.1007/s12221-010-0455-4

Lai, Ch. G., Gao, H. H., Zhai, Y. F., Chen, H., Liu, Q., Dang, H. Y., Yu, Y., \& Zheng, F. Q. (2018). Effect of sublethal concentrations of three pesticides on the activity of detoxifying enzymes in Drosophila melanogaster and Drosophila suzukii. Journal of Environmental Entomology. 40(3): 676-683.

Lee, S. O., Jeon, J. M., Oh, C. W., Kim, Y. M., Kang, C. K., Lee, D. S., Mykles, D. L., \& Kim, H. W. (2011). Two juvenile hormone esterase-like carboxylesterase cDNAs from a Pandalus shrimp (Pandalopsis japonica): cloning, tissue expression, and effects of eyestalk ablation. Comparative Biochemistry and Physiology Part B: Biochemistry and Molecular Biology. 159, 148-156. http// doi: 10.1016/j.cbpb.2011.03.004 .

Li, X., Schuler, M. A., \& Berenbaum, M. R. (2007). Molecular mechanisms of metabolic resistance to synthetic and natural xenobiotics. Annual Review of Entomology. 52, 
231-253.

http//doi:10.1146/annurev.ento.51.110104.151104

Lima, A. V. B. (2013). Characterization of esterase patterns in hepatopancreas of three species of Macrobrachium (Palaemonidae). Biochem. Syst. Ecol. 47, 132-138. http// doi: 10.1016/ j.bse.2012.10.006

Livak, K J. (2008). Analyzing real-time PCR data by the comparative Ct method. Nature Protocol, 3 (6), 11011108.

http://www.nature.com/doifinder/10.1038/nprot.2008 .73

Liu, S., Feng, M. F. \& He, M. Z. (2015). Identification and expression profiles of Cnapha- locrocis medinalis craboxylesterase genes. Chinese Journal of Applied Entomology. 52 (3), 662-670.

Oakeshott, J. G., Claudianos, C., Campbell, P. M., Newcomb, R. D. \& Russell R. J. (2005). Biochemical genetics and genomics of insect esterases. In: Gilbert, L.I., latrou, K., Gill, S.S.(Eds), Comp. Mol.Insect Sci. Elsevier, Oxford, pp. 309-381. http// doi: 10.1016/ B0-44-451924-6/00073-9

Ren, N. N., Xie, M., You, Y. Ch. \& You M. Sh. (2014). An overview on the study of insect carboxylesterases (COEs) and the COE-mediated resistance to insecticides. Journal of Fujian Agriculture and Forestry University (Natural Science Edition). 43 (3), 337-334.

Shen, H., Ma, Y., \& Hu, Y. (2015). Near-full-length genome sequence of a novel reovirus from the Chinese mitten $\mathrm{crab}, \backslash \mathrm{r}$, eriocheir sinensis. Genome Announcements, 3(3), e00447-15.

https://www.ncbi.nlm.nih.gov/pmc/articles/PMC44409 49/

Shen, H. Sh., Zang, Y. N., Song, K., Ma, Y. Ch., Dai, T. H. \& Ali S. (2017). A meta-transcriptomics survey reveals changes in the microbiota of the Chinese mitten crab Eriocheir sinensis infected with hepatopancreatic necrosis disease. Frontiers in Microbiology.8, 732-739. http// doi: 10.3389/ fmicb. 2017. 00732

Sin, Y. W., Kenny, N. J., Qu, Z., Chan, K. W., Cheong, S. P., Leunq, R.W., Chan, T. F., Bendena, W. G., Chu, K. H., Tobe, S. S. \& Hui, J.H. (2015). Identification of putative ecdysteroid and juvenile hormone pathway genes in the shrimp Neocaridina denticulate. General and Comparative Endocrinology. 214, 167-176.

Tao, T., Xie, X., Liu, M., Jiang, Q., \& Zhu, D. (2017). Cloning of two carboxylesterase cdnas from the swimming crab portunus trituberculatus: molecular evidences for their putative roles in methyl farnesotae degradation. Comparative Biochemistry \& Physiology Part B Biochemistry \& Molecular Biology, 203, 100-107. http//doi:10.1016/j.cbpb.2016.10.001

Tatjana, T., Nevenka, K. (2006). Abamectin in the aquatic environment. Ecotoxicology. 15(6): 495-502. http://doi:10.1007/s10646-006-0085-1

Taylor, K. P. \& Radic, M. Z. (1994). The cholinesterases: from genes to proteins. Annu Rev Pharmacol Toxicol, 34(34), 281-320.http// doi:10.1146/ annurev.pa. 34. 040194. 001433

Teng, X. \& Sun, M. J. (2003). Advanees on carboxylesterase researeh. Chinese Bulletiu of Life Sciences,15, 31-35.

Thomas, B., Church, W. T., \& Hammock, B. (2015). Homology model of juvenile hormone esterase from the crop pest, heliothis virescens. Proteins-structure Function \& Bioinformatics, 34(2), 184-196.

Wendt-Rasch, L., Pirzadeh, P., Woin, P. (2003). Effects of metsulfuron methyl and cypermethrin exposure on freshwater model ecosystems. Aquatic Toxicology. 63, 243-256. http://doi:10.1016/S0166-445X(02)00183-2 http//doi:10.1002/(SICI)10970134(19990201)34:2<184::AID-PROT4>3.0.CO;2-8 •

$\mathrm{Xu}$, Ch. \& Liu, Y. (2017). Accumlation and elimination of deltamethrin in Megalobramaa amblycephala. Journal of Northwest A\&F University (Natural Science Edition). 45(12): 31-37.

Xu, Y., Zhao, M., Deng, Y., Yang, Y., Li, X., Lu, Q., Ge, J., Pan, J. $\& X u, Z$. (2017). Molecular cloning, characterization and expression analysis of two juvenile hormone esteraselike carboxylesterase cdnas in Chinese mitten crab, Eriocheir sinensis. Comparative Biochemistry \& Physiology Part B Biochemistry \& Molecular Biology, 205, 46-53. https://doi.org/10.1016/j.fsi.2015.04.022

Zhang, M. W., Liu, Y. \& Li, H. (2012). The research progress of Microbial Esterases. Journal of Guangdong University of Education. 32 (3): 66-71.

Zhang, Z. W. (2014). Cloning and Functional Analysis of Carboxylesterase Gene Bmbe2 in the Silkworm, Bombyx mori (MD Thesis). Chongqing University, Chongqing, China.

Zhang, J., Li, D., Ge, P., Guo, Y., Zhu, K. Y., \& Ma, E., (2014). Molecular and functional characterization of cdnas putatively encoding carboxylesterases from the migratory locust, locusta migratoria. Plos One, 9(4), e94809. http//doi:10.1371/journal. pone.0094809 